\title{
Analysis of DAR(1)/D/s Queue with Quasi-Negative Binomial-II as Marginal Distribution
}

\author{
Kanichukattu Korakutty Jose ${ }^{1}$, Bindu Abraham ${ }^{2}$ \\ ${ }^{1}$ Department of Statistics, St. Thomas College Palai Mahatma Gandhi University, Kottayam, India \\ ${ }^{2}$ Department of Statistics, Baselios Poulose II Catholicose College, \\ Mahatma Gandhi University, Kottayam, India \\ E-mail:kkjstc@gmail.com,babpc@rediffmail.com \\ Received March 5, 2011; revised June 13, 2011; accepted June 20, 2011
}

\begin{abstract}
In this paper we consider the arrival process of a multiserver queue governed by a discrete autoregressive process of order 1 [DAR(1)] with Quasi-Negative Binomial Distribution-II as the marginal distribution. This discrete time multiserver queueing system with autoregressive arrivals is more suitable for modeling the Asynchronous Transfer Mode(ATM) multiplexer queue with Variable Bit Rate (VBR) coded teleconference traffic. DAR(1) is described by a few parameters and it is easy to match the probability distribution and the decay rate of the autocorrelation function with those of measured real traffic. For this queueing system we obtained the stationary distribution of the system size and the waiting time distribution of an arbitrary packet with the help of matrix analytic methods and the theory of Markov regenerative processes. Also we consider negative binomial distribution, generalized Poisson distribution, Borel-Tanner distribution defined by Frank and Melvin(1960) and zero truncated generalized Poisson distribution as the special cases of Quasi-Negative Binomial Distribution-II. Finally, we developed computer programmes for the simulation and empirical study of the effect of autocorrelation function of input traffic on the stationary distribution of the system size as well as waiting time of an arbitrary packet. The model is applied to a real data of number of customers waiting for checkout in an airport and it is established that the model well suits this data.
\end{abstract}

Keywords: Discrete Autoregressive Process of Order [DAR(1)], Multiserver ATM Multiplexer, Matrix Analytic Methods, Markov Renewal Process, Markov Regenerative Theory, Teleconference Traffic, Quasi-Negative Binomial Distribution-II, Generalized Poisson Distribution, Borel-Tanner Distribution

\section{Introduction}

In B-ISDN/ATM network, IP packets or cells of voice, video, data are sent over a common transmission channel on statistical multiplexing basis. The performance analysis of statistical multiplexer whose input consists of a superposition of several packetized sources is not a straightforward one. The difficulty in modeling this type of traffic is due to the correlated structure of arrivals. A common approach is to approximate this complex non renewal input process by analytically tractable arrival process, namely discrete autoregressive process (DAR). The impact of autocorrelation in traffic processes on queueing performance measures such as mean queue length, mean waiting times and loss probabilities in finite buffers, can be very dramatic.
The DAR process, constructed and analyzed by Jacobs and Lewis [1] has developed into one of several standard tools for modelling input traffic in telecommunication networks. The discrete autoregressive process of order 1 [DAR(1)] is known to be a good model for VBR coded teleconference traffic as in Elwalid et al. [2]. Kamoun and Ali [3] modeled an ATM multiplexer as a discrete time multiserver queueing system with on-off sources and studied the transient and stationary distribution of the number of packets in the system.

Hwang and Sohraby [4] obtained the closed form expression for the stationary probability generating function of the system size of the discrete time single server queue with DAR(1) input. Hwang et al. [5] obtained the waiting time distribution of the discrete time single server queue with DAR(1) input. Choi and Kim [6] analyzed a 
multiserver queue fed by DAR(1) input. Kim et.al [7] derived mean queue size in a queue with discrete autoregressive arrival of order $p$.

In this paper we analyzed a discrete-time multi-server queue with s servers $(s>0)$ having deterministic service times (specifically, service time is 1) and the following arrival process: Let $A_{m}$ be the number of arrivals at time $m=0,1,2, \cdots$. Then $A_{m}=A_{m-1}$ with probability $\beta$; otherwise, $A_{m}$ is sampled independently from a quasi-negative binomial distribution-II. The stationary distribution of the waiting time in that queue is calculated numerically with a matrix analytic method. Specifically, the arrival process is first analyzed at embedded times when $A_{m}$ is sampled independently of $A_{m-1}$ or when $A_{m}$ is less than the number of servers. This analysis reduces to an analysis of a Markov chain of $\mathrm{M} / \mathrm{G} / 1$ type as presented in Neuts [8]. Then the stationary distribution of $A_{m}$ at general $m$ is derived, which in turn gives the stationary distribution of the waiting time.

The rest of the paper is arranged as follows. QuasiNegative Binomial Distribution-II is described in Section 2. Queues with input traffic as DAR(1) with marginal Quasi-Negative Binomial-II is explained in Section 3. Analysis of DAR(1) /D/s queue with marginal QuasiNegative Binomial Distribution-II is given in Section 4. The stationary distribution of the Markov renewal process is given in Section 5. Deriving the stationary distribution of system size and waiting time of an arbitrary packet is explained in Sections 6 and 7. The quantitative effect of the stationary distribution of system size and waiting time on the autocorrelation function as well as the parameters of the input traffic is illustrated numerically in Section 8. The application to real data set is given in Section 9.

\section{Quasi Negative Binomial Distribution-II}

The quasi-negative binomial distribution (QNBD) obtained by Janardan [9], Sen and Jain [10] has the probability mass function as

$$
\begin{aligned}
& P\left(x ; n, p_{1}, p_{2}\right)=\frac{(n+x-1) ! p_{1}\left(p_{1}+x p_{2}\right)^{x-1}}{(n-1) ! x !\left(1+p_{1}+x p_{2}\right)^{x+n}} \\
& \text { for }\left(p_{1}+x p_{2}\right) \geq 0 ; n>0, p_{1}>0, \text { if } p_{2}>0 \\
& \text { and }\left(p_{1}+x p_{2}\right) \geq 0 ; \text { if } p_{2}<0 ; x=0,1,2 \cdots
\end{aligned}
$$

where $x$ be the number of occurrences. When $p_{2}=0$ the QNBD reduces to negative binomial distribution (NBD) and when $n=1$, QNBD reduces to quasi geometric distribution (QGSD) for $n=1$. QNBD tends to the Consul and Jain's [11] generalized Poisson distribution. But unfortunately the moments of this distribution appear in an infinite series which is not suitable for summation. The method of moments fails to provide quick estimates of its parameters. Hence Ahmad et al. [12] introduced a new model of quasi negative binomial distribution-II (QNBDII). This new model has the probability mass function

$$
p(x)=\frac{\left(n p_{2}-p_{1}-1\right)}{\left(n p_{2}-1\right)}\left(\begin{array}{c}
n+x-1 \\
x
\end{array}\right) \frac{p_{1}\left(p_{1}+x p_{2}\right)^{x-1}}{\left(1+p_{1}+x p_{2}\right)^{x+n+1}}
$$

for $0<n p_{2}<1$ and $0<p_{1}<1 ; x=0,1,2 \cdots$

When $p_{2}=0$ this new model reduces to negative binomial distribution. The probabilities of QNBD-II decreases with the successive occurrences. This tendency of probabilities suggests its possible applications in reliability, biometry, and survival analysis. The QNBD-II is uni-model and only its first moment (mean) appears in compact form. The lower and upper bound of Mode $M$ is

$$
\begin{gathered}
\frac{n p_{1}-1}{1-p_{1}}<M<\frac{p_{1}(n-1)}{1-p_{1}}, p_{1}<1 . \\
\text { Mean }=\frac{n p_{1}}{\left(1-n p_{2}\right)}, p_{2}<1
\end{gathered}
$$

\subsection{Remarks}

1) Let $X$ be a quasi-negative binomial variate with parameters $\left(n, p_{1}, p_{2}\right)$ and pmf given by (2). If $n \Rightarrow \infty$ such that $n p_{1}=\alpha$ and $n p_{2}=\lambda$ then the random variable $X$ tends to generalized Poisson distribution with parameters $(\alpha, \lambda)$.

2) Let $X$ be a quasi-negative binomial variate with parameters $\left(n, p_{1}, p_{2}\right)$ and probability mass function is (pmf) is given by (2). If $n \Rightarrow \infty$ such that $n \lambda^{-1}=\alpha$ where $\lambda^{-1}=p_{2}$ then the random variable $X$ tends to Borel-Tanner distribution defined by Frank and Melvin [13]

3) Let $X$ be a quasi-negative binomial variate with parameters (n, $\left.p_{1}, p_{2}\right)$ and pmf given by (2), then zerotruncated quasi-negative-binomial distribution-II tends to zero-truncated generalized Poisson distribution as $n \Rightarrow \infty$.

\section{Queues with DAR(1) Arrivals with Quasi Negative Binomial Distribution-II as Marginal}

The input ATM multiplexer with VBR coded teleconference traffic is assumed to be DAR(1) with quasinegative binomial distribution-II as marginal. Let $\{Y(t): t=0,1,2 \cdots\}$ be a sequence of i.i.d random variables. $\mathrm{Y}(\mathrm{t})$ assumes positive values only and $b_{x}=P[Y(t)=x], x=0,1,2, \cdots$. When the input process has quasi-negative binomial distribution-II as marginal we have $b_{x}$ as the pmf of the form (2). 
Discrete Autoregressive Process of order 1 (DAR(1) $\{X(t): t=0,1,2, \cdots\}$ is defined by the regression equation as

$$
\begin{aligned}
& X(0)=Y(0) \\
& X(t)=(1-Z(t)) X(t-1)+Z(t) Y(t), t=1,2, \cdots
\end{aligned}
$$

where $\{Z(t): t=1,2,3, \cdots\}$ are i.i.d Bernoulli random variables with $P[Z(t)=0]=\beta(0 \leq \beta<1)$ and $P[Z(t)=1]=1-\beta$ and $\{Z(t): t=1,2,3, \cdots\}$ is assumed to be independent of $\{Y(t): t=0,1,2, \cdots\}$. $\operatorname{DAR}(1)$ is determined by the parameter $\beta$ and the distribution $\left\{b_{x}: x=0,1,2, \cdots\right\}$ of $Y(t)$, so that

$$
\begin{gathered}
X(0)=Y(0) \\
X(t)= \begin{cases}X(t-1) & \text { with prob. } \beta \\
Y(t) & \text { with prob. } 1-\beta\end{cases}
\end{gathered}
$$

The properties of DAR(1) are as follows

1) $\{X(t): t=0,1,2 \cdots\}$ is stationary

2) The probability distribution of $X(t)$ is the same as the distribution of $\mathrm{Y}(\mathrm{t})$

$$
P[X(t)=x]=b_{x}, x=0,1,2 \cdots
$$

3) The autocorrelation function for $X(t)$ at lag $t$ is obtained as

$$
\gamma(t)=\frac{\operatorname{Cov}(X(0)) ; X(t))}{\operatorname{Var}(X(0))}=\beta^{t}, t=0,1,2 \cdots
$$

the parameter $\beta$ is the decay rate of the autocorrelation function.

\section{Analysis of DAR(1)/D/s Queue with Quasi-Negative Binomial-II as Marginal}

We assume that the input process is $\operatorname{DAR}(1)$ with quasi-negative binomial distribution-II distribution as the marginal distribution and there are $s$ servers $(s>0)$ whose service occurs at constant rate. In this integer valued time queue, the time is divided into slots of equal size and one slot is needed to serve a packet by a server. We assume that packet arrivals occur at the beginning of slots and departures occur at the end of the slots. Here $\{X(t): t=0,1,2 \cdots\}$ represents packet arrivals so that $X(t)$ is the number of packets arriving at the beginning of the $t^{\text {th }}$ slot.

Let $N(t)$ be the number of packets in the system say system size, immediately before arrivals at the beginning of the $t^{\text {th }}$ slot. Then

$\{(N(t), X(t)): t=0,1,2 \cdots\}$ is a two dimensional Markov process of $\mathrm{M} / \mathrm{G} / 1$ queue type. The state space is

$$
\bigcup_{n \geq 0} l(n)=\bigcup_{n, i \geq 0}\{(n, i)\}=E\{0,1,2, \cdots\} \times\{0,1,2 \cdots\}
$$

The number of phases is infinity. So the computation of stationary distribution of $\{(N(t), X(t)): t=0,1,2 \cdots\}$ is not easy to work out.

In practice by matrix analytical method and using the theory Markov regenerative processes, we compute the stationary distribution of the new process at the embedded epochs $\left\{t_{\tau}, \tau=0,1,2 \cdots\right\}$

$0<t_{0}<t_{1}<t_{2}<t_{3} \cdots$ as follows, we have

$$
\begin{aligned}
& t_{\tau}=0, \tau=0 \\
& =\inf \left\{\mathrm{t}>\mathrm{t}_{\tau-1}: \mathrm{Z}(t)=1 \text { or } 0 \leq X(t) \leq s-1\right\}, \tau=1,2 \cdots
\end{aligned}
$$

Let

$$
\begin{gathered}
N_{\tau}=N\left(t_{\tau}\right), \tau=0,1,2 \cdots \\
J_{0}=s \\
J_{\tau}=\left\{\begin{array}{lll}
X\left(t_{\tau}\right) & \text { if } Z\left(t_{\tau}\right)=0 & \tau=1,2,3 \cdots \\
s & \text { if } Z\left(t_{\tau}\right)=1 & \tau=1,2,3 \cdots
\end{array}\right.
\end{gathered}
$$

The packet arrivals at and after $t_{\tau}$ are independent of the information prior to $t_{\tau}$ given $J_{\tau}$. From this, it is observed that $\left\{\left(N_{\tau}, J_{\tau}\right): \tau=0,1,2, \cdots\right\}$ is the new Markov renewal process with state space

$$
E=\{0,1,2 \cdots\} \times\{0,1 \cdots s\}
$$

The probability transition matrix of the Markov renewal process is computed as follows.

1) For $n=0,1,2 \cdots$ and $i=0,1, \cdots, s-1$

$$
(n, i) \rightarrow \begin{cases}(\max \{n-s+i, 0\}, i) & \text { with prob. } \beta \\ (\max \{n-s+i, 0\}, s) & \text { with prob. } 1-\beta\end{cases}
$$

2) For $n=0,1,2 \ldots$

$$
(n, s) \rightarrow\left\{\begin{array}{l}
(\max \{n-s+i, 0\}, i) \text { with prob. } \\
\quad b_{i} \beta 0 \leq i \leq s-1, \\
(n-s+i, s) \quad \text { with prob. } \\
b_{i}(1-\beta) s-n+1 \leq i \leq s-1, \\
(0, s) \quad \text { with prob. } \sum_{i=0}^{\min \{s-n, s-1\}} b_{i}(1-\beta)+g_{0} \delta_{n 0} \\
(n+l, s) \quad \text { with prob. } g_{l} l \geq 0, n+l>0
\end{array}\right.
$$

where

$$
\begin{gathered}
\delta_{n 0}= \begin{cases}1 & \text { if } n=0 \\
0 & \text { if } n \geq 1\end{cases} \\
g_{0}=b_{s} \\
g_{l}=\sum_{i \mid l} b_{i+s}(1-\beta) \beta^{\frac{l}{i}-1}, l=1,2, \cdots
\end{gathered}
$$

The transition probability matrix $P$ 


$$
\left(\begin{array}{cccccc}
B_{s} & A_{s+1} & \cdots & A_{2 s-1} & A_{2 s} & \cdots \\
B_{s-1} & A_{s} & \cdots & A_{2 s-2} & A_{2 s-1} & \cdots \\
\vdots & \vdots & \vdots & \vdots & \vdots & \cdots \\
B_{1} & A_{2} & \cdots & A_{c} & A_{s+1} & \cdots \\
A_{0} & A_{1} & \cdots & A_{s-1} & A_{s} & \cdots \\
0 & A_{0} & \cdots & A_{s-2} & A_{s-1} & \cdots \\
0 & 0 & \cdots & A_{s-3} & A_{s-2} & \cdots \\
\vdots & \vdots & \vdots & \vdots & \vdots & \\
0 & 0 & \cdots & 0 & 0 & \cdots
\end{array}\right)
$$

is obtained as above.

Where the elementary matrices are

$$
\begin{aligned}
& \begin{array}{llllll}
0 & \cdots & & i & \cdots & s
\end{array}
\end{aligned}
$$

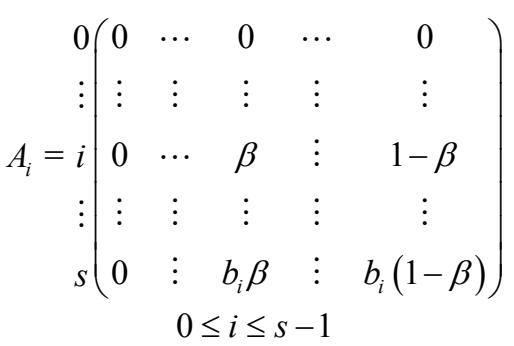

$$
\begin{aligned}
& \begin{array}{lll}
0 & \cdots & s
\end{array}
\end{aligned}
$$

$$
\begin{aligned}
A_{i}= & \vdots\left(\begin{array}{ccc}
0 & \cdots & 0 \\
\vdots & \vdots & \vdots \\
0 & \cdots & g_{i-s}
\end{array}\right) i \geq s, \\
& B_{i}=\sum_{j=0}^{i} A_{j}, 1 \leq i \leq s
\end{aligned}
$$

We assume that the stability condition $\lambda=E[X(t)]=\sum_{m=1}^{\infty} m b_{m}<s$ is satisfied.

\section{The Stationary Distribution of the Markov Renewal Process}

Consider $\left\{\left(N_{\tau}, J_{\tau}\right), \tau=0,1,2 \cdots\right\}$, and $\pi_{n i}=\lim _{\tau \rightarrow \infty} P\left\{N_{\tau}=n, J_{\tau}=i\right\}, n \geq 0,0 \leq i \leq s$ We apply matrix ${ }^{\tau \rightarrow \infty}$ analytic method as described below. The transition probability matrix $P$ has infinite order, so that it would have to be truncated before we implement matrix analytic method. We assume that there exists some index $\mathrm{N}$ such that $A_{N}=0$ for all $n>N$. That is we assume that the Markov chain does not jump more than $\mathrm{N}$ steps at a time so that the matrix is of finite order, see Latouche and Ramaswamy [14]. For a numerical illustration, consider the case when $\mathrm{s}=5$ and $\mathrm{N}=14$. Then the transition probability matrix $P$ can be obtained as




By arranging the transition probability matrix into (sxs) matrices we get

$$
P=\left(\begin{array}{ccc}
\hat{B}_{0} & \hat{B}_{1} & \hat{B}_{2} \\
\hat{A}_{0} & \hat{A}_{1} & \hat{A}_{2} \\
0 & \hat{A}_{0} & \hat{A}_{1} \\
0 & 0 & \hat{A}_{0}
\end{array}\right)
$$

or equivalently

$$
P=\left(\begin{array}{ccc}
\hat{B}_{0} & \hat{A}_{2} & \hat{A}_{3} \\
\hat{A}_{0} & \hat{A}_{1} & \hat{A}_{2} \\
0 & \hat{A}_{0} & \hat{A}_{1} \\
0 & 0 & \hat{A}_{0}
\end{array}\right)
$$

In general we can symbolize the transition matrix $P$ as

$$
\begin{aligned}
P= & \left(\begin{array}{ccccc}
\hat{B}_{0} & \hat{B}_{1} & \hat{B}_{2} & \cdots & \hat{B}_{n^{*}-1} \\
\hat{A}_{0} & \hat{A}_{1} & \hat{A}_{2} & \cdots & \hat{A}_{n^{*}-1} \\
0 & \hat{A}_{0} & \hat{A}_{1} & \cdots & \hat{A}_{n^{*}-2} \\
\vdots & \vdots & \vdots & \vdots & \vdots \\
0 & 0 & 0 & \cdots & \hat{A}_{0}
\end{array}\right) \\
n^{*}=\frac{N+s+1}{s}-1 &
\end{aligned}
$$

or equivalently

$$
P=\left(\begin{array}{ccccc}
\hat{B}_{0} & \hat{A}_{2} & \hat{A}_{3} & \cdots & \hat{A}_{n^{*}} \\
\hat{A}_{0} & \hat{A}_{1} & \hat{A}_{2} & \cdots & \hat{A}_{n^{*}-1} \\
0 & \hat{A}_{0} & \hat{A}_{1} & \cdots & \hat{A}_{n^{*}-2} \\
\vdots & \vdots & \vdots & \vdots & \vdots \\
0 & 0 & 0 & \cdots & \hat{A}_{0}
\end{array}\right)
$$

The elements of $\mathrm{P}$ can be written as

$$
\begin{gathered}
\hat{B}_{0}=\left(\begin{array}{cccc}
B_{s} & A_{s+1} & \cdots & A_{2 s-1} \\
B_{s-1} & A_{s} & \cdots & A_{2 s-2} \\
\vdots & \vdots & \vdots & \vdots \\
B_{1} & A_{2} & \cdots & A_{s}
\end{array}\right), \\
\hat{A}_{n}=\left(\begin{array}{cccc}
A_{s n} & A_{s n+1} & \cdots & A_{s(n+1)-1} \\
A_{s n-1} & A_{s n} & \cdots & A_{s(n+1)-2} \\
\vdots & \vdots & \vdots & \vdots \\
A_{s(n-1)+1} & A_{s(n-1)+2} & \cdots & A_{s n}
\end{array}\right) \\
n=0,1,2 \cdots n^{*}, \\
\hat{B}_{n}=\hat{A}_{n+1}, n=1,2, \cdots n^{*}
\end{gathered}
$$

A matrix $P$ of the above structure is said to be of M/G/1 type, which underlines the similarity to the embedded Markov chain of the M/G/1 queue. With respect to the levels, the Markov chain is called skip free to the left, since in one transition the level can be reduced only by one.

By the matrix analytic method we proceed as follows.

Step 1: Find the minimal nonnegative solution $G$ of the matrix equation

$$
G=\sum_{n=0}^{\infty} \hat{A}_{n} G^{n}
$$

$G$ can be given by the following iteration See Breuer [15]

$$
\begin{aligned}
G_{0} & =0 \\
G_{1} & =\hat{A}_{0} \\
G_{k} & =\sum_{n=1}^{k-1} \hat{A}_{n} G_{k-1}^{n}, k=2,3, \cdots \\
G & =\sum_{k=1}^{\infty} G_{k}
\end{aligned}
$$

$G$ is a stochastic matrix , and hence we can stop the iteration procedure when $|1-G .1|<\varepsilon$ reaches where $\varepsilon=0.0001$. From this iteration we obtained the upper limit of $\mathrm{k} \&$ let $n^{*}=k-1$. From this $n^{*}$ we come to know the truncated index $\mathrm{N}$ at which $G$ becomes stochastic

Step 2: Find

$$
H=\sum_{n=0}^{n^{*}} \hat{B}_{n} G^{n}
$$

and a positive row vector h satisfying $h H=h$

Step 3:

$$
\begin{aligned}
x_{0}= & h \\
x_{n}= & \left(x_{0} \sum_{i=0}^{n^{*}} \hat{B}_{n+i} G^{i}+\sum_{l=1}^{n-1} x_{l} \sum_{i=0}^{n^{*}} \hat{A}_{n-l+i+1} G^{i}\right) \\
& \times\left(I-\sum_{i=0}^{n^{*}} \hat{A}_{i+1} G^{i}\right)^{-1}, n=1,2 \cdots n^{*}
\end{aligned}
$$

Step 4: Finally

$$
\begin{aligned}
& \left(\left(\pi_{n s, 0}, \cdots \pi_{n s, s}\right) \cdots\left(\pi_{(n+1) s-1,0} \cdots \pi_{(n+1) s-1, s}\right)\right)=C x_{n}, \\
& n=0,1,2, \cdots, n^{*}
\end{aligned}
$$

where $C=\left[\sum_{n=0}^{n^{*}} x_{n} \mathrm{e}\right]^{-1}$ and $e$ is the $s(s+1)$ dimensional column vector whose components are all ones.

\section{Stationary Distribution of $\{N(t), X(t), t=0,1,2 \cdots\}$}

Observe that $\left\{\left(\left(N_{\tau}, J_{\tau}\right), t_{\tau}\right), \tau=0,1,2 \ldots\right\}$ is a Markov 
renewal process and

$\left\{\left(N\left(t+t_{\tau}\right), X\left(t+t_{\tau}\right)\right): t=0,1,2 \cdots\right\}$ given

$\left\{\left((N(v), X(v)), 0 \leq v<t_{\tau}, N_{\tau}, J_{\tau}\right)=(n, i)\right\}$ is

stochastically equivalent to $\{N(t), X(t): t=0,1,2 \cdots\}$

given $\left\{N_{0}, J_{0}=(n, i)\right\}$. Hence

$\{N(t), X(t): t=0,1,2 \cdots\}$ is a discrete time Markov regenerative process with the Markov renewal sequence $\left\{\left(\left(N_{\tau}, J_{\tau}\right), t_{\tau}\right): \tau=0,1,2 \cdots\right\}$. From the theorm See Kulkarni [16]

$p_{n j}=\lim _{t \rightarrow \infty} P\{(N(t), X(t))=(n, j)\} n, j=0,1,2 \cdots$ of $\{(N(t), X(t)): t=0,1,2 \cdots\}$ are determined by

$$
p_{n j}=\frac{\sum_{l=0}^{\infty} \sum_{i=0}^{s} \pi_{l i} E\left[\sum_{t=t}{ }^{t_{\tau+1}-1} 1_{(N(t), X(t))=(n, j)} \mid\left(N_{\tau}, J_{\tau}\right)=(l, i)\right]}{\sum_{l=0}^{\infty} \sum_{i=0}^{s} \pi_{l i} E\left[t_{\tau+1}-t_{\tau} \mid\left(N_{\tau}, J_{\tau}\right)=(l, i)\right]}
$$

We have

$$
\begin{gathered}
E\left[\sum_{t_{\tau+t_{\tau}} t_{\tau+1^{-1}} 1_{\{(N(t), X(t))=(n, j)\}} \mid} \mid\left(N_{\tau}, J_{\tau}\right)=(l, i)\right] \\
= \begin{cases}1 & \text { if } i=j, 0 \leq i \leq s-1 \text { and } n=l \\
b_{j} & \text { if } i=s, 0 \leq j \leq s-1 \text { and } n=l \\
\frac{b_{s}}{1-\beta} & \text { if } i=s, j=s \text { and } n=l \\
b_{j} \beta^{\frac{n-l}{j-s}} & \text { if } i=s, j>s, n \geq l \\
0 & \text { and } j-s \text { divides } n-l\end{cases}
\end{gathered}
$$

The numerator of Equation (3) is

$$
\begin{cases}\pi_{n j}+\pi_{n s} b_{j}, & 0 \leq j \leq s-1 \\ \frac{\pi_{n s} b_{s}}{1-\beta}, & j=s \\ \sum_{i=0}^{\left|\frac{n}{j-s}\right|} \pi_{n-i(j-s), s} b_{j} \beta^{i}, & j \geq s+1\end{cases}
$$

We have

$$
\begin{aligned}
& E\left[t_{\tau+1}-t_{\tau} \mid\left(N_{\tau}, J_{\tau}\right)=(l, i)\right] \\
& = \begin{cases}1 & \text { if } 0 \leq i \leq s-1, \\
\sum_{r=0}^{s-1} b_{r}+\sum_{r=s}^{\infty} b_{r} \frac{1}{1-\beta} & \text { if } i=s\end{cases}
\end{aligned}
$$

The denominator of Equation (3) is

$$
\sum_{l=0}^{\infty} \sum_{i=0}^{s-1} \pi_{l i}+\sum_{l=0}^{\infty} \pi_{l s}\left(\sum_{r=0}^{s-1} b_{r}+\sum_{r=s}^{\infty} b_{r} \frac{1}{1-\beta}\right)
$$

where $\left(\sum_{l=0}^{\infty} \pi_{l 0} \cdots \sum_{l=0}^{n^{*}} \pi_{l s}\right)$ is the stationary probability vector of the Markov process $\left\{J_{\tau}: \tau=0,1,2 \cdots\right\}$ whose transition probability matrix is

$$
\begin{aligned}
& \left(P\left(J_{\tau+1}=j \mid J_{\tau}=i\right)\right)_{0 \leq i, j \leq s} \\
& =\left(\begin{array}{ccccc}
\beta & 0 & \cdots & 0 & 1-\beta \\
0 & \beta & \cdots & 0 & 1-\beta \\
\vdots & \vdots & \vdots & \vdots & \vdots \\
0 & 0 & \cdots & \beta & 1-\beta \\
\beta b_{0} & \beta b_{1} & \cdots & \beta b_{s-1} & 1-\beta \sum_{r=0}^{s-1} b_{r}
\end{array}\right)
\end{aligned}
$$

The infinitesimal transition matrix of (5) is

$$
Q=\left(\begin{array}{cccc}
-(1-\beta) & 0 & \cdots & -(1-\beta) \\
0 & -(1-\beta) & \cdots & -(1-\beta) \\
\vdots & \vdots & \vdots & \vdots \\
0 & 0 & \cdots & -(1-\beta) \\
\beta b_{0} & \beta b_{1} & \cdots & -\beta \sum_{r=0}^{s-1} b_{r}
\end{array}\right)
$$

The balance equations are

$$
\prod Q=0 \& \prod \mathrm{e}=1
$$

By solving the balance equations we obtain the stationary distribution of the Markov process $\left\{J_{\tau}: \tau=0,1,2 \cdots\right\}$ as

$$
\sum_{l=0}^{\infty} \pi_{l i}= \begin{cases}\frac{\beta b_{i}}{1-\beta \sum_{r=s}^{\infty} b_{r}}, & 0 \leq j \leq s-1 \\ \frac{1-\beta}{1-\beta \sum_{r=s}^{\infty} b_{r}}, & i=s\end{cases}
$$

By substituting (6) into (4) we obtain the denominator of the right hand side of (3) as $\left(1-\beta \sum_{r=s}^{\infty} b_{r}\right)$

Theorem 6.1

The stationary distribution or the limiting probabilities

$$
p_{n j}=\lim _{t \rightarrow \infty} P\{(N(t), X(t))=(n, j)\}, n, j=0,1,2 \cdots
$$

are given by

$$
p_{n j}= \begin{cases}\mu^{-1}\left(\pi_{n j}+\pi_{n s} b_{j}\right), & 0 \leq j \leq s-1 \\ \mu^{-1} \frac{\pi_{n s} b_{s}}{1-\beta}, & j=s \\ \mu^{-1} \sum_{i=0}^{\left|\frac{n}{j-s}\right|} \pi_{n-i(j-s), s} b_{j} \beta^{i}, & j \geq s+1\end{cases}
$$

where $\left(1-\beta \sum_{r=s}^{\infty} b_{r}\right)^{-1}=\mu$ 


\section{Stationary Distribution of Waiting Time of an Arbitrary Packet}

Let $W$ denote the waiting time of an arbitrary packet at steady state. Then for $w=0,1,2 \cdots$.

$P(W=w)=$ (Mean number of arrivals in a slot at steady state whose waiting time is $w$ )/(Mean number of arrivals in a slot)

Suppose that there are $\mathrm{n}$ packets immediately before arrivals at the beginning of the $t^{\text {th }}$ slot and that the number of packet arrivals is $\mathrm{j}$ at the beginning of the $t^{\text {th }}$ slot, so that $N(t)=\mathrm{n}$ and $X(t)=j$. Then the number of packets whose waiting time is $\mathrm{w}$ among the ones who arrive at the beginning of the $t^{\text {th }}$ slot is

$$
\begin{cases}\min \{s(w+1)-n, j\}, & s w<n<s(w+1) \\ \min \{n=j-s w, s\}, & n \leq s w \leq n=j \\ 0 & \text { otherwise }\end{cases}
$$

Therefore the mean number of arrivals in a slot at steady state whose waiting time $\mathrm{w}$ is

$$
\begin{aligned}
& \sum_{n=0}^{s w} \sum_{j=s w-n+1}^{\infty} p_{n j} \min \{n=j-s w, s\} \\
& +\sum_{n=s w+1}^{s(w+1)-1} \sum_{j=1}^{\infty} p_{n j} \min \{s(w+1)-n, j\}
\end{aligned}
$$

Since the mean number of arrivals in a slot is $\lambda$, the following theorem is obtained from (7).

\section{Theorem 7.1}

The distribution of the waiting time $W$ of an arbitrary packet is given by

$$
\begin{aligned}
& P(W=w)=\frac{1}{\lambda}\left(\sum_{n=0}^{s w} \sum_{j=s w-n+1}^{\infty} p_{n j} \min \{n=j-s w, s\}\right. \\
& +\sum_{n=s w+1}^{s(w+1)-1} \sum_{j=1}^{\infty} p_{n j} \min \{s(w+1)-n, j\},
\end{aligned}
$$

\section{Empirical Study}

The complementary distribution function of the stationary system size when when $\lambda=2.5$ and $\beta=0.3,0.5,0.7$ $\& 0.9$ and the complementary distribution function of the stationary system size when $\beta=0.3$ and $p_{1}=0.009$, $0.0015,0.02 \& 0.024\left(p_{2}=0.0064,0004,0.002 \& 0.0004\right)$ respectively are derived.

The parameter $\beta$ gives the information on the strength of correlation of the input process. Stationary system size is larger for the large $\beta$ (see Figure 1). Also stationary system size stochastically increases when the parameter $p_{1}$ of the input process decreases (see Figure 2).

The complementary distribution function of the waiting time of an arbitrary packet, when $\lambda=2.5$ and $\beta=0.3$,

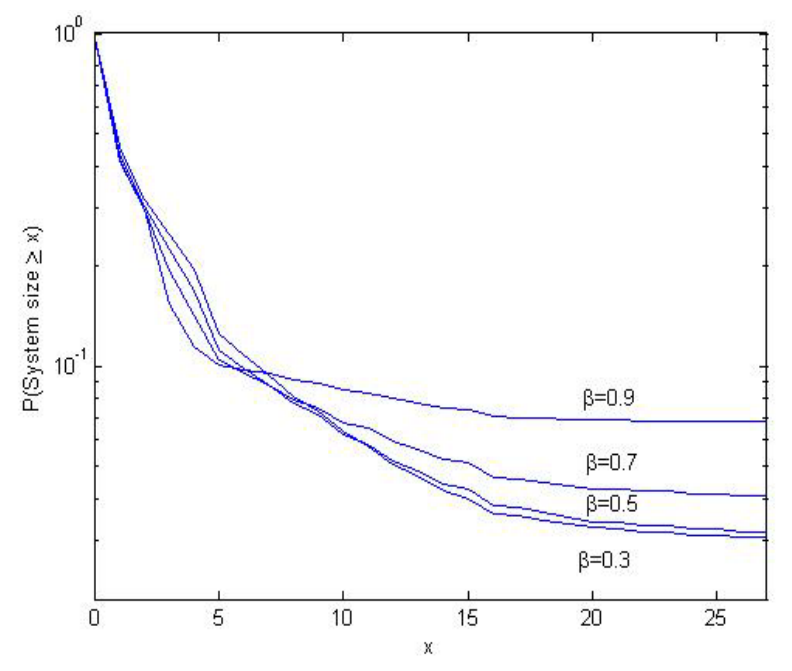

Figure 1. Complementary distribution function of the stationary system size, when $p_{1}=0.0045, p_{2}=0.0082, \lambda=2.5$.

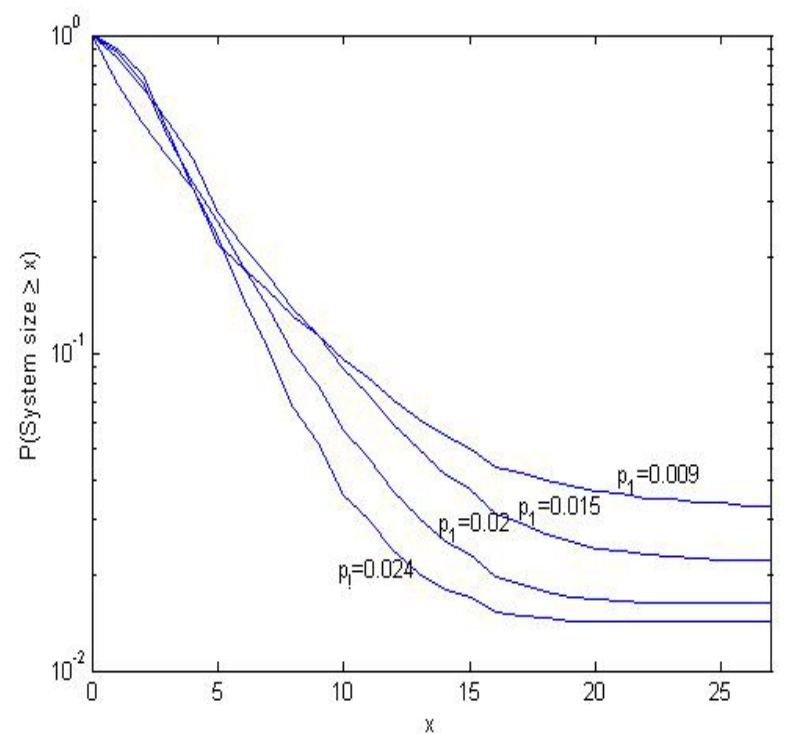

Figure 2. Complementary distribution function of the stationary system size, when $\lambda=2.5, \beta=0.3$.

$0.5,0.7 \& 0.9$ and the complementary distribution function of the waiting time when $\beta=0.3$ and $p_{1}=0.009$, $0.0015,0.02 \& 0.024\left(p_{2}=0.0064,0004,0.002 \& 0.0004\right)$ respectively are derived.

Stationary waiting time of an arbitrary packet, is larger for large $\beta$ (see Figure 3). Also stationary waiting time of an arbitrary packet, stochastically increases when the input parameter $p_{1}$ decreases (see Figure 4). We assume the number of servers to be 3

Tables 1-3 display the stationary probabilities of the system size for different values of $p_{1}, p_{2}, \& \beta$.

Tables $\mathbf{4}$ and $\mathbf{5}$ display the stationary probabilities of waiting time of an arbitrary packet for different values of $p_{1} \& \beta$. 


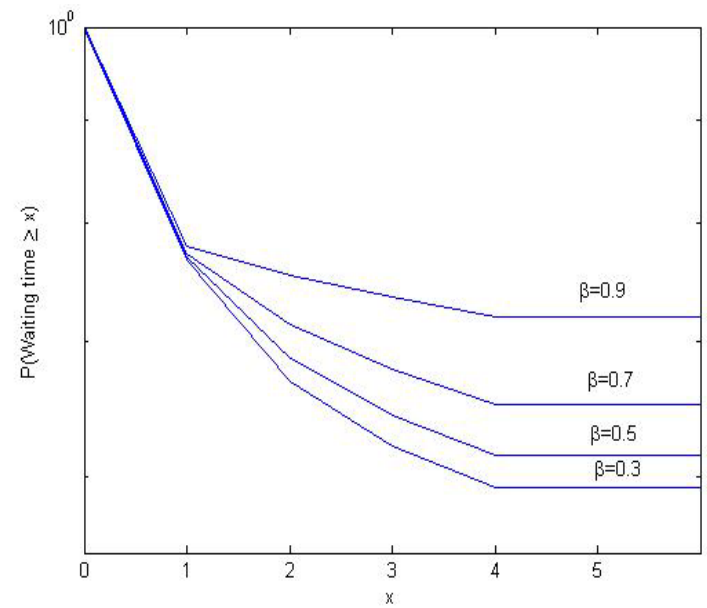

Figure 3. Complementary distribution function of the waiting time of an arbitrary packet, when $p_{1}=0.009, p_{2}=\mathbf{0 . 0 0 6 4}$.

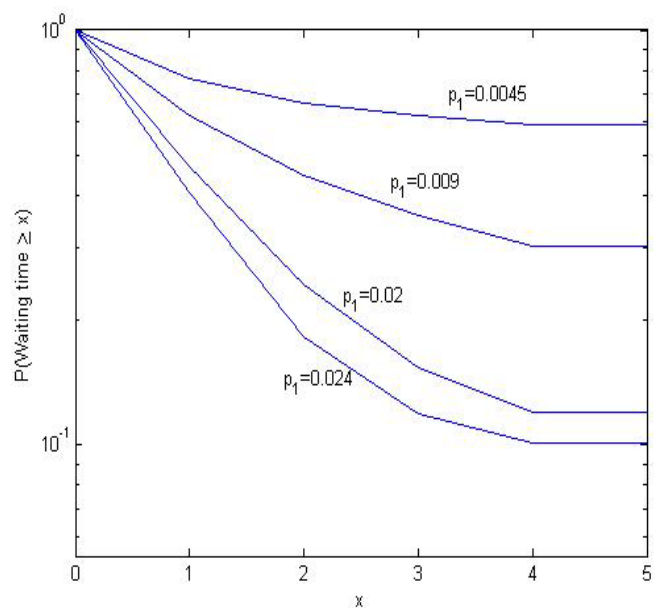

Figure 4. Complementary distribution function of the waiting time of an arbitrary packet, when $\beta=0.3$.

Table 1. Showing the values of distribution of stationary system $\operatorname{size} P(n, j)$ for $\lambda=2.5, \beta=0.1, p_{1}=0.0045, p_{2}=0.0082$ and $s=3$.

\begin{tabular}{|c|c|c|c|c|c|c|c|c|c|c|}
\hline \multicolumn{11}{|c|}{$j$} \\
\hline$n$ & 0 & 1 & 2 & 3 & 4 & 5 & 6 & 7 & 8 & $\ldots$ \\
\hline 0 & 0.5148 & 0.1005 & 0.0460 & 0.0264 & 0.0158 & 0.0114 & 0.0086 & 0.0067 & 0.0054 & $\ldots$ \\
\hline 1 & 0.0460 & 0.0090 & 0.0090 & 0.0026 & 0.0031 & 0.0011 & 0.0008 & 0.0006 & 0.0005 & $\cdots$ \\
\hline 2 & 0.0135 & 0.0026 & 0.0495 & 0.0007 & 0.0007 & 0.0014 & 0.0002 & 0.0001 & 0.0001 & ... \\
\hline 3 & 0.0104 & 0.0021 & 0.0009 & 0.0005 & 0.0004 & 0.0003 & 0.0010 & 0.0001 & 0.0001 & $\ldots$ \\
\hline 4 & 0.0081 & 0.0016 & 0.0007 & 0.0004 & 0.0003 & 0.0003 & 0.0002 & 0.0007 & 9.E - 04 & $\ldots$ \\
\hline 5 & 0.0005 & 0.0012 & 0.0005 & 0.0003 & 0.0002 & 0.0001 & 0.0001 & 0.0001 & 0.0006 & ... \\
\hline 6 & 0.0043 & 0.0009 & 0.0004 & 0.0002 & 0.0001 & 0.0001 & 0.0001 & 8.E-04 & 0.0001 & $\cdots$ \\
\hline 7 & 0.0034 & 0.0006 & 0.0003 & 0.0001 & 0.0001 & 0.0001 & 8.E - 04 & 6.E-04 & 5.E - 04 & \\
\hline 8 & 0.0028 & 0.0005 & 0.0002 & 0.0001 & 0.0001 & 8.E-04 & 6.E - 04 & 0.0001 & 4.E - 04 & $\ldots$ \\
\hline 9 & 0.0028 & 0.0005 & 0.0002 & 0.0001 & 0.0001 & 8.E-04 & 7.E - 04 & 5.E - 04 & 4.E - 04 & $\ldots$ \\
\hline 10 & 0.0023 & 0.0004 & 0.0002 & 0.0001 & 9.E - 04 & 6.E-04 & 5.E - 04 & 4.E - 04 & 8.E - 04 & \\
\hline 11 & 0.0019 & 0.0003 & .00017 & 00011 & 7.E - 04 & 5.E-04 & 4.E - 04 & 3.E - 04 & 3.E - 04 & \\
\hline$\vdots$ & $\vdots$ & & $\vdots$ & $\vdots$ & $\vdots$ & $\vdots$ & $\vdots$ & $\vdots$ & $\vdots$ & ?. \\
\hline
\end{tabular}

Table 2. Showing the values of distribution of stationary system size $P(n, j)$ for $\lambda=2.5, \beta=0.3, p_{1}=0.009, p_{2}=0.0094$ and $s=3$.

\begin{tabular}{|c|c|c|c|c|c|c|c|c|c|c|}
\hline \multicolumn{11}{|c|}{$j$} \\
\hline$n$ & 0 & 1 & 2 & 3 & 4 & 5 & 6 & 7 & 8 & $\ldots$ \\
\hline 0 & 0.5875 & 0.1148 & 0.1413 & 0.0300 & 0.0020 & 0.0014 & 0.0010 & 0.0008 & 0.0006 & $\cdots$ \\
\hline 1 & 0.0009 & 0.0001 & 0.0100 & 0.0004 & 0.0018 & 2.E - 04 & 1.E - 04 & 1.E - 04 & 1.E - 04 & $\ldots$ \\
\hline 2 & 0.0097 & 0.0046 & 0.0682 & 00206 & 0.0040 & 0.0054 & 0.0005 & 0.0003 & 0.0003 & $\ldots$ \\
\hline 3 & 0.0001 & 3.E - 05 & 2.E - 05 & 4.E - 05 & 0.0014 & 2.E - 05 & 0.0009 & 1.E - 05 & $9 . \mathrm{E}-05$ & $\ldots$ \\
\hline 4 & 8.E - 05 & 1.E - 05 & 1.E - 05 & 3.E - 05 & 0.0013 & 0.0011 & 1.E - 05 & 0.0007 & 7.E - 05 & $\ldots$ \\
\hline 5 & 2.E - 05 & 1.E - 05 & 7.E - 05 & 2.E - 05 & 0.0012 & 2.E - 05 & 2.E - 05 & 1.E - 05 & 0.0006 & $\ldots$ \\
\hline 6 & 3.E - 05 & 1.E - 05 & 8.E - 05 & 2.E - 05 & 0.0010 & 0.0010 & 0.0008 & 1.E - 05 & 9.E - 05 & $\ldots$ \\
\hline 7 & 3.E - 05 & 6.E - 06 & 5.E - 06 & 1.E - 05 & 0.0009 & 1.E - 05 & 1.E - 05 & 1.E - 06 & 1.E - 06 & $\ldots$ \\
\hline 8 & 3.E - 05 & 6.E - 06 & 5.E - 06 & 1.E - 05 & 0.0009 & 1.E - 05 & 1.E - 05 & 1.E - 06 & 1.E - 06 & $\ldots$ \\
\hline 9 & 0.0016 & 0.0007 & 0.0005 & 0.0003 & 0.0002 & 0.0002 & 0.0002 & 0.0001 & 0.0007 & $\ldots$ \\
\hline 10 & 2.E - 05 & 4.E - 06 & 3.E - 06 & 1.E - 05 & 0.0007 & 0.0008 & 1.E - 05 & 2.E - 06 & 0.0005 & $\ldots$ \\
\hline 11 & 1.E - 05 & 3.E - 06 & 1.E - 06 & 9.E - 06 & 0.0006 & 1.E - 05 & 2.E - 06 & 1.E - 06 & 8.E - 06 & $\ldots$ \\
\hline$\vdots$ & $\vdots$ & $\vdots$ & $\vdots$ & $\vdots$ & $\vdots$ & $\vdots$ & $\vdots$ & $\vdots$ & $\vdots$ & $\ddots$. \\
\hline
\end{tabular}


Table 3. Showing the values of distribution of stationary system $\operatorname{size} P(n, j)$ for $\lambda=2.5, \beta=0.3, p_{1}=0.024, p_{2}=0.0004$ and $s=3$.

\begin{tabular}{|c|c|c|c|c|c|c|c|c|c|c|}
\hline \multicolumn{11}{|c|}{$j$} \\
\hline$n$ & 0 & 1 & 2 & 3 & 4 & 5 & 6 & 7 & 8 & $\ldots$ \\
\hline 0 & 0.0922 & 0.1530 & 0.1759 & 0.1358 & 0.0605 & 0.0320 & 0.0147 & 0.0060 & 0.0022 & $\ldots$ \\
\hline 1 & 0.0064 & 0.0150 & 0.0208 & 0.0190 & 0.0266 & 0.0040 & 0.0020 & 0.0008 & 0.0003 & $\ldots$ \\
\hline 2 & 0.0603 & 0.0089 & 0.0111 & 0.0115 & 0.0131 & 0.0120 & 0.0012 & 0.0005 & 0.0001 & $\ldots$ \\
\hline 3 & 0.0024 & 0.0060 & 0.0077 & 0.0073 & 0.0072 & 0.0030 & 0.0052 & 0.0003 & 0.0001 & $\ldots$ \\
\hline 4 & 0.0013 & 0.0032 & 0.0044 & 0.0041 & 0.0040 & 0.0040 & 0.0010 & 0.0019 & 6.E - 05 & $\ldots$ \\
\hline 5 & 0.0001 & 0.0017 & 0.0021 & 0.0022 & 0.0021 & 0.0010 & 0.0006 & 0.0003 & 0.0007 & $\ldots$ \\
\hline 6 & 0.0004 & 0.0010 & 0.0013 & 0.0013 & 0.0012 & 0.0010 & 0.0017 & 0.0002 & 0.0001 & $\ldots$ \\
\hline 7 & 0.0002 & 0.0005 & 0.0007 & 0.0007 & 0.0006 & 0.0001 & 0.0004 & 0.0001 & $6 . \mathrm{E}-05$ & $\ldots$ \\
\hline 8 & 0.0001 & 0.0002 & 0.0003 & 0.0003 & 0.0003 & 0.0004 & 0.0002 & 0.0006 & 4.E - 05 & $\ldots$ \\
\hline 9 & 7.E - 05 & 0.0001 & 0.0002 & 0.0002 & 0.0002 & 0.0002 & 0.0005 & 0.0001 & 4.E - 05 & $\ldots$ \\
\hline 10 & 4.E - 05 & 9.E - 05 & 0.0001 & 0.0001 & 0.0001 & 0.0002 & 0.0001 & 6.E - 05 & 0.0002 & $\ldots$ \\
\hline 11 & 2.E - 05 & 4.E - 05 & 6.E - 05 & 6.E - 05 & $6 . E-05$ & 8.E - 05 & 7.E - 05 & 4.E - 05 & 3.E - 05 & $\ldots$ \\
\hline$\vdots$ & $\vdots$ & $\vdots$ & $\vdots$ & $\vdots$ & $\vdots$ & $\vdots$ & $\vdots$ & $\vdots$ & $\vdots$ & $\ddots$ \\
\hline
\end{tabular}

Table 4. Showing the values of the distribution of waiting time of an arbitrary packet $P(W=\omega)$ for different values of $\beta$ and $\lambda$ $=2.5, p_{1}=0.009, p_{2}=0.0064$ and $s=3$.

\begin{tabular}{|c|c|c|c|c|c|}
\hline \multicolumn{6}{|c|}{$\beta$} \\
\hline$\omega$ & 0.1 & 0.3 & 0.5 & 0.7 & 0.9 \\
\hline 0 & 0.2332 & 0.2326 & 0.2306 & 0.2270 & 0.2211 \\
\hline 1 & 0.1096 & 0.0991 & 0.0832 & 0.0598 & 0.0245 \\
\hline 2 & 0.0499 & 0.0473 & 0.0432 & 0.0359 & 0.0187 \\
\hline 3 & 0.0280 & 0.0289 & 0.0285 & 0.0257 & 0.0158 \\
\hline$\vdots$ & $\vdots$ & $\vdots$ & $\vdots$ & $\vdots$ & $\ddots$ \\
\hline
\end{tabular}

Table 5. Showing the values of the distribution of waiting time of an arbitrary packet $P(W=\omega)$ for different values of $p_{1}, \beta=$ 0.3 , and $s=3$.

\begin{tabular}{|c|c|c|c|c|c|}
\hline \multicolumn{6}{|c|}{$p_{2}$} \\
\hline & 0.0082 & 0.0064 & 0.004 & 0.002 & 0.0004 \\
\hline \multicolumn{6}{|c|}{$p_{1}$} \\
\hline$\omega$ & 0.0045 & 0.009 & 0.015 & 0.02 & 0.024 \\
\hline 0 & 0.2326 & 0.3748 & 0.4847 & 0.5303 & 0.5944 \\
\hline 1 & 0.0991 & 0.1790 & 0.2407 & 0.2267 & 0.2229 \\
\hline 2 & 0.0473 & 0.0898 & 0.1102 & 0.0890 & 0.0636 \\
\hline 3 & 0.0289 & 0.0543 & 0.0569 & 0.0341 & 0.0179 \\
\hline$\vdots$ & $\vdots$ & $\vdots$ & $\vdots$ & $\vdots$ & $\ddots$ \\
\hline
\end{tabular}

\section{Analysis and Modeling of a Data Set}

In this section we apply the model to a data on the number of initially waiting customers for checking in an airport for a time period of 30 minutes each

$\{t=0,1, \cdots, 30\}$. The data was collected from morning 8.00 A.M to 11.30 P.M for one week. This includes all the busy periods as well as idle periods. The data is taken from the file customer checkout.xlsx available in [17]. Table 6 gives the frequency distribution of the corre- sponding data, where $x$ is the number of customers initially waiting for the service.

In the present paper we assumed the number of arrivals as DAR(1) with marginal Quasi Negative Binomial II distribution. Thus the data set can be fitted to the the Quasi Negative Binomial II distribution as follows.

To test whether there is a significant difference between an observed distribution and the Quasi Negative Binomial II distribution, we use Kolmogorov-Smirnov [K.S.] test for $H_{0}$ : Quasi Negative Binomial II distri- 
Table 6. Table showing the frequency distribution of the number of customers waiting for checkout.

\begin{tabular}{cccc}
\hline$x$ & frequency & $x$ & frequency \\
\hline 0 & 43 & 12 & 8 \\
1 & 49 & 13 & 2 \\
2 & 47 & 14 & 5 \\
3 & 44 & 15 & 3 \\
4 & 29 & 16 & 3 \\
5 & 22 & 17 & 2 \\
6 & 30 & 18 & 0 \\
7 & 14 & 19 & 1 \\
8 & 15 & 20 & 0 \\
9 & 5 & 21 & 1 \\
10 & 8 & 22 & 1 \\
11 & 4 & Total & 336 \\
\hline
\end{tabular}

bution with parameter $p_{1}=0.021$ and $p_{2}=0.00513$ is a good fit for the given data. Here the calculated value of the K.S. test statistic is 0.017857 and the critical value corresponding to the significance level 0.01 is 0.088924 , showing that the assumption for number of arrivals follow Quasi Negative Binomial II distribution is valid (see Figure 5).

By applying matrix analytic method we obtain the stationary distribution of system size and waiting time of an arbitrary customer for the Quasi Negative Binomial $\mathrm{II} / \mathrm{D} / \mathrm{s}$ queue. Here the mean $=\lambda=4.3125$. To satisfy the stability condition we assume the number of servers as $s=5$. Also we assume the value of autocorrelation function $\beta=0.1, p_{1}=0.021$ and $p_{2}=0.00513$. Tables 7 and 8 display the stationary distribution of waiting time of an arbitrary customer and system size.

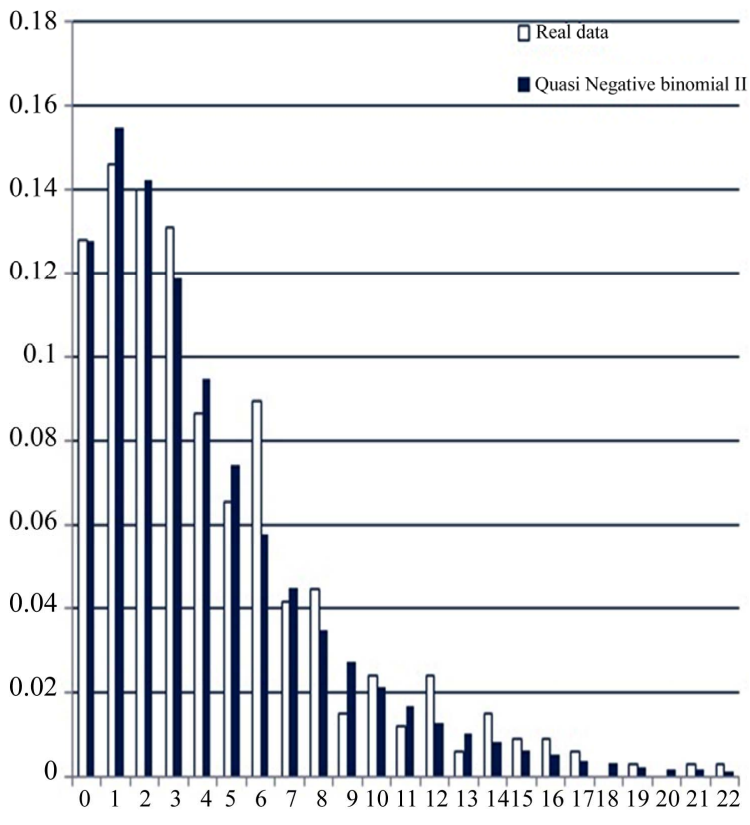

Figure 5. The Probability histogram of real data and the Quasi Negative Binomial II distribution with $p_{1}=0.021$ and $p_{2}=0.00513$.

Table 7. Table showing the stationary distribution of waiting time of an arbitrary customer $P(W=\omega)$ when $\beta=0.1, \lambda=$ 4.3125, $s=5$.

\begin{tabular}{cc}
\hline$w$ & $p(w)$ \\
\hline 0 & 0.2832 \\
1 & 0.1195 \\
2 & 0.0589 \\
3 & 0.0380 \\
$\vdots$ & $\vdots$ \\
\hline
\end{tabular}

Table 8. Table showing the stationary distribution of system $\operatorname{size} P(n, j)$ when $\beta=0.1, \lambda=4.3125, s=5$.

\begin{tabular}{cccccccccccccc}
$n$ & 0 & 1 & 2 & 3 & 4 & 5 & 6 & 7 & 8 & 9 & $\ldots$ \\
\hline 0 & 0.102 & 0.123 & 0.114 & 0.094 & 0.075 & 0.058 & 0.041 & 0.031 & 0.024 & 0.019 & $\ldots$ \\
1 & 0.007 & 0.009 & 0.008 & 0.008 & 0.008 & 0.004 & 0.003 & 0.002 & 0.001 & 0.001 & $\ldots$ \\
2 & 0.005 & 0.006 & 0.007 & 0.006 & 0.007 & 0.003 & 0.002 & 0.001 & 0.001 & 0.001 & $\ldots$ \\
3 & 0.004 & 0.005 & 0.005 & 0.005 & 0.005 & 0.003 & 0.002 & 0.001 & 0.001 & 0.001 & $\ldots$ \\
4 & 0.004 & 0.005 & 0.005 & 0.005 & 0.005 & 0.003 & 0.002 & 0.001 & 0.001 & 0.001 & $\ldots$ \\
5 & 0.003 & 0.003 & 0.003 & 0.003 & 0.003 & 0.002 & 0.001 & 0.031 & 0.000 & 0.000 & $\ldots$ \\
6 & 0.002 & 0.003 & 0.003 & 0.003 & 0.003 & 0.001 & 0.001 & 0.000 & 0.000 & 0.000 & $\ldots$ \\
7 & 0.002 & 0.002 & 0.002 & 0.002 & 0.002 & 0.0033 & 0.000 & 0.000 & 0.000 & 0.000 & $\ldots$ \\
8 & 0.001 & 0.001 & 0.001 & 0.001 & 0.001 & 0.001 & 0.000 & 0.000 & 0.000 & 0.000 & $\ldots$ \\
9 & 0.001 & 0.001 & 0.001 & 0.001 & 0.001 & 0.001 & 0.007 & 0.000 & 0.000 & 0.000 & $\ldots$ \\
$\vdots$ & $\vdots$ & $\vdots$ & $\vdots$ & $\vdots$ & $\vdots$ & $\vdots$ & $\vdots$ & $\vdots$ & $\vdots$ & $\vdots$ & $\ddots$ \\
\hline
\end{tabular}




\section{Conclusions}

In this paper we analyze DAR(1)/D/s queue with QuasiNegative Binomial Distribution-II as the marginal distribution. Based on the matrix analytic methods and by using the theory of Markov regenerative processes, we obtained the stationary distributions of the system size and the waiting time of an arbitrary packet. From the definition of autocorrelation function we can say that the larger the parameter $\beta$, the slower the decay of the autocorrelation of the input process. So it is expected that stationary system size and waiting time for the case of large $\beta$ are stochastically larger than those for the case of small $\beta$. Also the stationary system size and waiting time increases when the input parameter $p_{1}$ decreases.

\section{References}

[1] P. A. Jacobs and P. A.W. Lewis, "Discrete Time Series generated by Mixtures III: Autoregressive Processes (DAR(p))," Naval Postgraduate School, Monterey, 1978.

[2] A. Elwalid, D. Heyman, T. V. Laksman, D. Mitra and A. Weiss, "Fundamental Bounds and Approximations for ATM Multiplexers with Applications to Video Teleconferencing," IEEE Journal of Selected Areas in Communications, Vol. 13, No. 6, 1995, pp. 1004-1016. doi:10.1109/49.400656

[3] F. Kamoun and M. M. Ali, "A New Theortical Approach for the Transient and Steady-State Analysis of Multiserver ATM Multiplexers with Correlated Arrivals," 1995 IEEE International Conference on Communications, Vol. 2, 1995, pp. 1127-1131. doi:10.1109/ICC.1995.524276

[4] G. U. Hwang and K. Sohraby, "On the Exact Analysis of a Discrete Time Queueing System with Autoregressive Inputs," Queueing Systems, Vol. 43, No. 1-2, 2003, pp. 29-41. doi:10.1023/A:1021848330183

[5] G. U. Hwang, B. D. Choi and J. K. Kim, "The Waiting Time Analysis of a Discrete Time Queue with Arrivals as an Autoregressive Process of Order 1," Journal of Ap- plied Probability, Vol. 39, No. 3, 2003, pp. 619-629.

[6] B. D. Choi, B. Kim, G. U. Hwang and J. K. Kim, "The Analysis of a Multiserver Queue Fed by a Discrete Autoregressissive Process of Order 1," Operations Research Letters, Vol. 32, No. 1, 2004, pp. 85-93. doi:10.1016/S0167-6377(03)00068-3

[7] J. Kim, B. Kim and K. Sohraby, "Mean Queue Size in a Queue with Discrete Autoregressive Arrivals of Order p,' Annals of Operations Research, Vol. 162, No. 1, 2008, pp. 69-68. doi:10.1007/s10479-008-0318-1

[8] M. F. Neuts, "Structured Stochastic Matrices of the M/G/1 Type and Their Applications," Dekker, New York. 1989.

[9] K. G. Janardhan, "Markov-Polya urn Model with PreDetermined Strategies," Gujarat Statistical Review, Vol. 2, No. 1, 1975, pp. 17-32.

[10] K. Sen and R. Jain "Generalized Markov-Polya urn Model Withpre-Determined Strategies," Journal of Statistical Planning and Inference, Vol. 54, 1996, pp. 119133. doi:10.1016/0378-3758(95)00161-1

[11] G. C. Jain and P. C. Consul, "A Generalized Negative Binomial Distribution," Society for Industrial Mathematics, Vol. 21, No. 4, 1971, pp. 501-513. doi:10.1137/0121056

[12] S. B. Ahmad, A. Hassan and M. J. Iqbal, "On a Quasi Negative Binomial Distribution-II and Its Applications," Preprint, 2010.

[13] A. H. Frank and A. B. Melvin, "The Borel-Tannerdistribution," Biometrica, Vol. 47, No. 1-2, 1960, pp. 143-150.

[14] G. Latouche and V. Ramaswamy, "Introduction to Matrix Analytical Method in Stochastic Modelling," Society for Industrial Mathematics, Pennsylvania, 1991.

[15] L. Breuer and D. Baum "An Introduction to Queueing Theory and Matrix Analytic Method," Springer, Berlin, 2004.

[16] V. G. Kulkarni "Modelling and Analysis of Stochastic Systems," Chapman \& Hall, London, 1995.

[17] Customer Check Out. xlsx http://www.westminstercollege.edu 